\title{
Utilization and perioperative complications of laparoscopic cryoablation vs. robotic partial nephrectomy for localized renal tumors
}

\author{
Aaron C. Weinberg ${ }^{1}$, Solomon L. Woldu ${ }^{1}$, Timothy Wen ${ }^{2}$, Christopher M. Deibert ${ }^{1}$, Ruslan Korets ${ }^{1}$, \\ Ketan K. Badani ${ }^{3}$ \\ ${ }^{1}$ Department of Urology, Columbia University College of Physicians and Surgeons, New York NY, USA; \\ ${ }^{2}$ Keck School of Medicine of the University of Southern California, Los Angeles, CA, USA; ${ }^{3}$ Department \\ of Urology, Mount Sinai Medical Center, New York, NY, USA
}

\section{ABSTRACT}

Objective: To compare the utilization, perioperative complications and predictors of LCA versus RPN in the treatment of localized renal tumors.

Methods: From the Nationwide Inpatient Sample we identified patients undergoing RPN or LCA for the treatment of localized renal tumors from October 2008 through 2010. Patient and hospital-specific factors which predict postoperative complications and use of LCA were investigated.

Results: 14,275 patients with localized renal tumors were identified: $70.3 \%$ had RPN and $29.7 \%$ had LCA. LCA was more common in older patient and at hospitals without robotic consoles. No difference was identified in perioperative complications $(0.2 \%$ vs. $0.2 \%$ ), transfusion (5.1\% vs. $6.2 \%$ ), length of stay (2.9 vs. 3.0 days) or median cost $(\$ 41,753$ vs. $\$ 44,618)$ between the groups, LCA vs. RPN. On multivariate analysis sicker patients were more likely to have LCA (OR 1.34, $\mathrm{p}=0.048$ ) and sicker patients had greater postoperative complications (OR 3.30, $\mathrm{p}<0.001$ ); LCA did not predict more complications (OR 1.63, $\mathrm{p}=0.138$ ) and LCA was performed at hospitals without RCs (OR 0.02, $\mathrm{p}<0.001$ ). Limitations include observational study design, inability to assess disease severity, operative time, or body mass index, which may affect patient selection and outcomes.

Conclusions: More patients had RPN vs. LCA; surgical technique was not predictive of postoperative complications. As technology develops to treat localized renal tumors, it will be important to continue to track outcomes and costs for procedures including RPN and LCA.

\section{ARTICLE INFO}

\section{Key words:}

Robotics; Kidney Neoplasms;

therapy [Subheading];

Cryosurgery

Int Braz J Urol. 2015; 41: 473-85

Submitted for publication:

June 17, 2014

Accepted after revision:

October 19, 2014

\section{INTRODUCTION}

In the last decade there has been a rising incidence of localized renal tumors largely related to the increasing use of cross-sectional abdominal imaging (1-3). These masses are often discovered incidentally by abdominal ultrasound or cross-sectional imaging and they are generally slow growing and detected at an earlier stage $(4,5)$. Management options can broadly be classified as active surveillance versus ablative or extirpative surgery. Nephron sparing (NS) approaches are increasing in utilization compared to the previous "gold standard" radical nephrectomies, because they offer equal oncologic and functional outcomes while preserving normal renal parenchyma 
(6). NS surgery for the localized renal tumors is now the recommended approach whenever possible by urological guidelines (7).

In recent years there has been a rising interest in minimally invasive treatment options. This includes laparoscopic and robotic assisted laparoscopic partial nephrectomy (LPN, RPN) and ablative therapies such as radiofrequency ablation (RFA) and cryoablation (CA). RPN has emerged as a viable alternative to mitigate the technical challenges of LPN, demonstrating perioperative outcomes that are comparable to LPN, with the benefit of reduced warm ischemia time (8).

A theoretical advantage to ablative techniques for localized renal tumors is that they represent a simpler operation, without a need for hilar dissection, clamping of renal vessels, do not result in kidney ischemia, and do not require a renorrhaphy to repair the renal defect. Of the ablative procedures available, cryoablation has a more predictable ablative zone and there is some evidence favoring cryotherapy over radiofrequency due to better surgical and oncological outcomes $(9,10)$. Laparoscopic assisted cryoablation (LCA) has been utilized in various tumor types in the kidney where the percutaneous approach may not be feasible. In this regard, LCA could be considered an alternative if perioperative complication rates and oncologic results are comparable to RPN.

In 2011 Klatte et al. performed a systematic review examining LCA and partial nephrectomy; specifically, the study focused on complications and oncologic outcomes of LCA, open partial nephrectomy (OPN) and LPN (11). After multivariable analysis LCA was found to have significantly worse oncologic outcomes with similar perioperative outcomes. Given that RPN now represents 70\% of all PN performed nationally and both LCA and RPN require general anesthesia, similar patient positioning and port placement, we sought to examine which patients would most benefit from LCA (12). In this study we explore the utilization and perioperative complications of LCA and RPN; additionally we examined factors which predicted the use of LCA in the treatment of patients with localized renal tumors, using a contemporary population-based cohort.

\section{MATERIALS AND METHODS}

\section{Data Source}

Data from the Nationwide Inpatient Sample (NIS) were abstracted from October 2008 until December 2010. The NIS includes all inpatient hospital discharge data collected via federal-state partnerships, as part of the Agency for Healthcare Research and Quality's Healthcare Cost and Utilization Project. NIS is a nationally representable database of hospitals within the United States, including public and private hospital as well as academic medical centers. The NIS is the only hospital database in the United States that collects charge information on all patients regardless of payer, including those patients covered by Medicare, Medicaid, private insurance, and the uninsured.

Sample Population and Surgical Procedures Using a previously described extraction methodology, all patients with a primary diagnosis code 189.0 of cancer of the kidney were identified using the 9th Revision of the International Classification of Disease, Clinical Modification (ICD-9-CM). Patients who underwent LCA (55.34) were abstracted. Patients who underwent PN (55.4) were also identified. Beginning October 1, 2008, the robot-assisted modifier (ICD-9-CM 17.4x) code was first utilized by the Centers for Medicare and Medicaid Services and the National Center of Health Statistics and was used to identify all RPN (this excluded pure laparoscopic PN) (13). We excluded patients $<18$ years old; with an admission type other than elective; and with diagnoses of transplant (v59.4), renal pelvis tumor (189.1), and pyelonephritis (590.00, 590.01, 590.10, 590.11).

To reduce the potential for confounding by tumor complexity, we initially excluded from analysis patients undergoing the following concomitant procedures: splenectomy $(41.4,41.42,41.43)$, liver resection $(50.2,50.21-26,50.29,50.3)$, pancreas resection $(52.5,52.51-53,52.59,52.6,52.7)$, bowel or colon resection (45.5, 45.50-52, 45.61-62, 45.7, 45.71, 45.73-76, 45.79, 45.8, 45.81-83), or thrombectomy with vascular reconstruction $(37.10,38.05,38.07$, 38.45 , 38.47, 38.65, 38.67, 38.75, 38.77, 38.87, 39.6, $39.61,39.63,39.66)$. This step resulted in exclusion of 58 patients, 28 LCA and 30 RPN. Because the NIS database does not have information on clinical sta- 
ging or pathologic data for these kidney cancers that were amenable to RPN or LCA, we will refer to these masses as localized renal tumors.

We also summed the total surgical procedures performed each year within individual hospitals and grouped each surgical procedure into hospital volume tertiles (low, medium and high); for RPN and LCA respectively $(<5,5-16,>16$ and $1,2-5,>5$ ). ristics

Baseline Patient and Hospital Characte-

Patient demographic characteristics including age at surgery, gender, and race (white, black and other races including: Hispanic, Asian, Pacific Islander, Native American, or other/unknown) were also examined. Secondary ICD-9 codes were used to define baseline Charlson Comorbidity Index (CCI) according to Charlson et al. (14) and adapted according to Deyo et al. (15). Patients were then stratified by CCI score as 0,1 , and $\geq 2$. To ensure uniformity of coding, detailed insurance categories were combined into more general categories including: private insurance, Medicare, Medicaid, and other (self-pay). Hospital characteristics included location (rural vs. urban) and region (Northeast, Midwest, South, West). The NIS does not provide any information on tumor size or staging for any cancer.

Intraoperative Complications and Postoperative Complications During Hospitalization

NIS records up to 15 diagnoses and procedures per each patient's in-hospital stay. Complications were identified using ICD-9 diagnoses 2 through 15. ICD-9 codes used for complications relied on previously published methodology; specifically seven groups of postoperative complications were identified, namely: Cardiac, Respiratory, Bowel, Genitourinary, Deep Venous Thromboembolism (DVT) and Pulmonary Embolism (PE), Infection, Hemorrhage and Wound (16). Additionally intraoperative complications were defined as any accidental puncture or laceration during a procedure. Blood transfusions were identified using the ICD-9 procedure for transfusion of homologous blood (99.04). In-hospital complications were defined as the presence of one or more complications.
Operative time, which may be related to peri-operative complications, is not a variable included in the NIS.

LOS, In-hospital Mortality and Costs

LOS is calculated by subtracting the admission date from the discharge date. In-hospital mortality information is abstracted from disposition of patient. We do not have capital cost data or cost of acquiring surgical systems and costs presented only include in-hospital total costs. All cost data was calculated from the cost to charge ratio as provided by the NIS. All total hospitalization costs were adjusted to 2013 U.S. dollars using the Consumer Price Index.

\section{Statistical analysis}

Descriptive statistics include estimated frequencies or proportions for categorical variables. Means, medians and ranges were reported for any continuously coded variable. Chi-square and independent-sample t-tests calculated the differences in, respectively, proportions and means.

Categorical distributions are reported as counts (\%) and continuous variables as means and standard deviations (SD), and medians and interquartile ranges (IQR). Fisher's exact test, Chi-square test, and Mantel-Haenszel test were used to assess differences in distribution among the categorical variables. The median test and t-test were used to assess the difference in distributions among continuous variables.

We fitted multi-level/mixed effects multivariable logistic regression models to test the associations of patient and hospital variables as predictors for performance of LCA and also for postoperative complications, while adjusting for clustering of patient covariates to the hospital level. All tests were two-sided with a statistical significance set at $\mathrm{p}<0.05$. All analyses were computed using SAS 9.3 (Cary, NC).

\section{RESULTS}

14,275 patients were identified from the NIS database from 2008-2010, of which 70.3\% $(10,034)$ had a RPN and 29.7\% $(4,421)$ had a LCA. Characteristics of the patient populations are presented in $\mathrm{Ta}-$ ble-1. Patient populations differed by race, insurance 


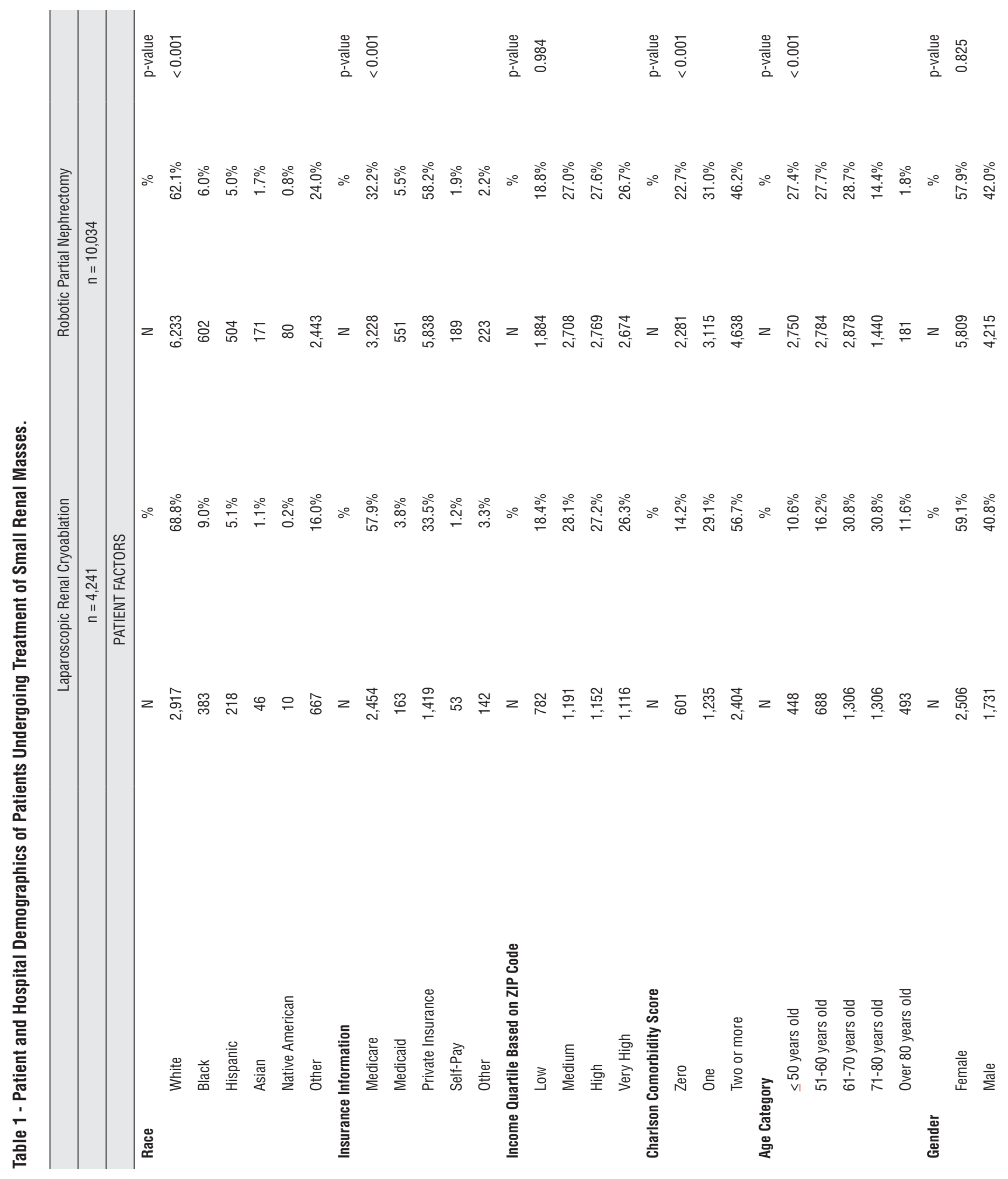




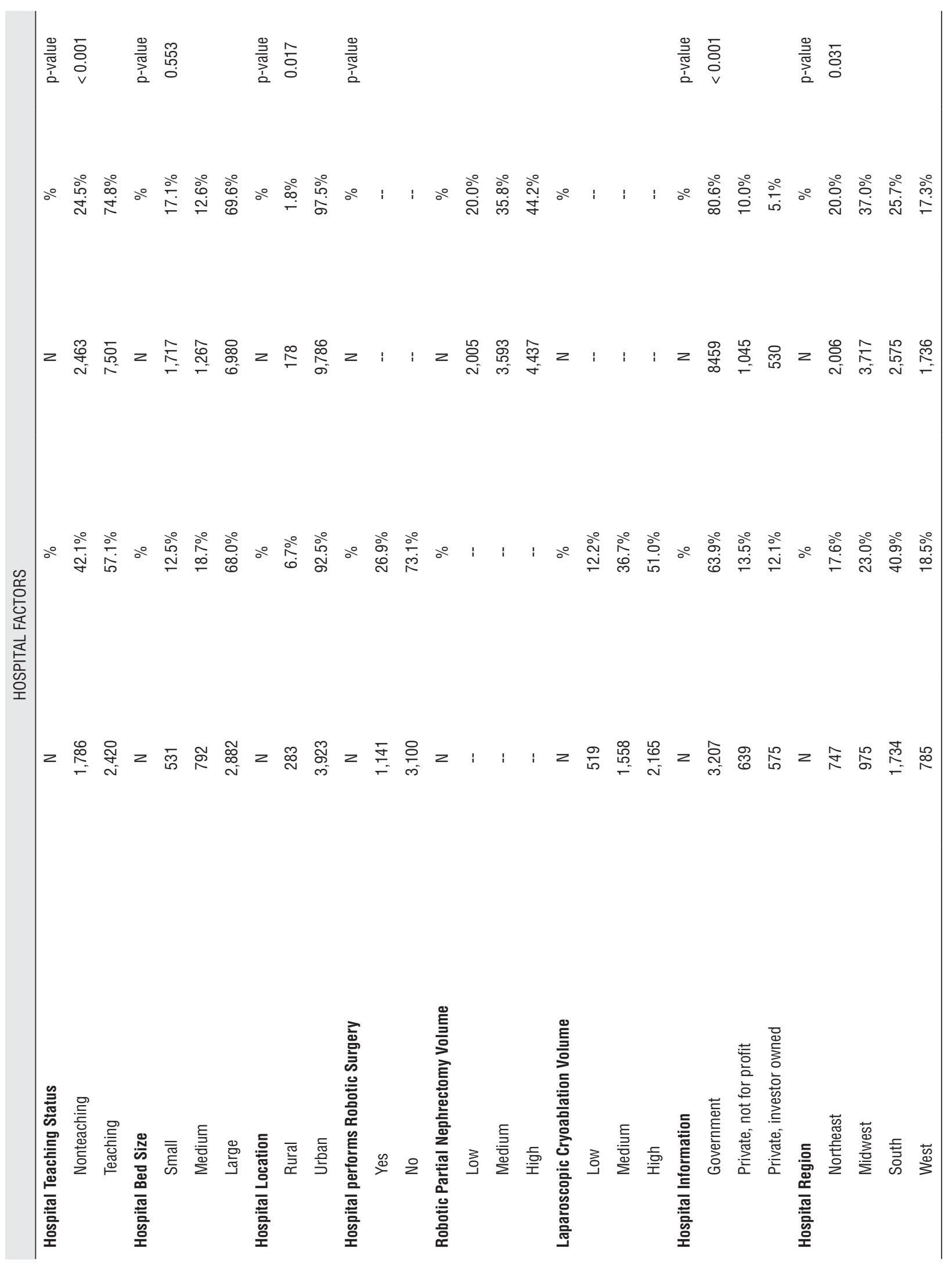


status, preoperative comorbidity, and age. A greater percentage of LCA patients were white $(68.8 \%$ vs. 62.1\%), had Medicare (57.9\% vs. 33.2\%), had a preoperative CCI score $\geq 2$ ( $56.7 \%$ vs. $46.2 \%$ ), and were $\geq 70$ years old ( $42.4 \%$ vs. $16.2 \%$ ). No difference was noted between groups when stratified by gender or income quartile. Hospital characteristics were significantly different between the two groups. Specifically, a higher proportion of RPN was performed at teaching institutions $(74.8 \%$ vs. $57.1 \%$ ) and government hospitals (80.6\% vs. 63.9\%). RPN was more often performed in the Midwest (37.0\%), whereas LCA was most commonly performed in the South (40.9\%). The largest percentage of each surgery was performed at high volume institutions (44.2\% and 51.0\%; LCA and RPN, $\mathrm{p}<0.001$ for both). Of note $73.1 \%$ of all LCA were performed at a hospital that does not perform robotic surgeries.

Table- 2 identifies the rate of intraoperative and postoperative outcomes, stratified by surgery. The rate of any complication occurring was found to be equivalent between $(10.14 \%$ vs. 9.92\%), as was the rate of blood transfusion (5.14\% vs. $6.15 \%)$, Length of Stay (LOS) $(2.89 \pm 0.18$ vs. $3.04 \pm 0.13$ ) and median cost ( $\$ 41,753$ vs. $\$ 44,618$; $\mathrm{p}=0.49$ ) for LCA vs. RPN respectively. However, statistically significant differences were noted for individual complications; specifically, patients undergoing RPN had lower rates of respiratory $(4.17 \%$ vs. $5.80 \%, p<0.001)$, infection $(2.06 \%$ vs. $4.07 \%, \mathrm{p}<0.001)$, and genitourinary complications (4.50\% vs. $3.87 \%, p=0.035)$.

On multivariate analysis (Table-3), patients were more likely to have their renal mass treated with LCA if they had multiple preoperative comorbidities compared to none (OR 1.34, 1.02$1.79 ; \mathrm{p}=0.048)$ or were from the highest income bracket vs. lowest (OR 1.58, 1.03-2.43; $\mathrm{p}=0.038$ ). Younger patients were significantly less likely to undergo LCA compared to older patients. Hospital factors that predicted LCA vs. RPN treatment in-

Table 2 - Complications by Surgical Approach for the Treatment of Small Renal Masses.

\begin{tabular}{lccc}
\hline & $\begin{array}{c}\text { Laparoscopic Renal } \\
\text { Cryoablation }\end{array}$ & Robotic Partial Nephrectomy & \\
\hline \% Complication & Overall & Overall & p-value \\
\hline Any & $10.14 \%$ & $9.92 \%$ & 0.139 \\
Infection & $4.07 \%$ & $2.06 \%$ & $<0.001$ \\
Respiratory & $5.80 \%$ & $4.17 \%$ & $<0.001$ \\
Genitourinary & $4.50 \%$ & $3.87 \%$ & 0.035 \\
Cardiac & $2.12 \%$ & $0.05 \%$ & 0.019 \\
DVT/PE & $1.29 \%$ & $0.67 \%$ & 0.364 \\
Bowel & $9.02 \%$ & $8.86 \%$ & 0.144 \\
Hemorrhage & $8.16 \%$ & $7.96 \%$ & 0.122 \\
Wound & $1.45 \%$ & $1.39 \%$ & 0.23 \\
$\quad$ Reoperation During Hospitalization & $3.77 \%$ & $2.49 \%$ & 0.466 \\
$\quad$ Intraoperative Intestinal Injury & $4.48 \%$ & $3.59 \%$ & 0.727 \\
$\quad$ Intraoperative Ureteral or Bladder Injury & $2.59 \%$ & $3.79 \%$ & 0.585 \\
$\quad$ Other Operative Injury & $3.33 \%$ & $2.79 \%$ & 0.301 \\
\% Blood Transfusion & $5.14 \%$ & $6.15 \%$ & 0.400 \\
Mean \pm SD LOS (days) & $2.89 \pm 0.18$ & $3.04 \pm 0.13$ & 0.610 \\
Median \$ costs (IQR) & $\$ 41,753$ & $\$ 44,618$ & 0.490 \\
& $(\$ 31,781-\$ 60,087)$ & $(\$ 33,548-\$ 60,532)$ & \\
\hline
\end{tabular}


cluded large hospital vs. small (OR 2.93, 1.07-8.03; $\mathrm{p}=0.037$ ), and LCA was less likely to be performed at hospitals that performed robotic surgeries (OR 0.02, 0.01-0.05; $\mathrm{p}<0.001$ ).

Additionally, patients with preoperative comorbidities were more likely to have a postoperative complication compared to patients without comorbidities (OR 1.63-3.30, $\mathrm{p}<0.001$ ) (Table-4). Interestingly, female gender was protective of complications (OR 0.77, 0.62-0.96; $p=0.017$ ); age, race and income were not predictors of post-operative complications. Regional variation existed with regard to complications and government hospitals, compared to private hospitals, were more likely to have complications (OR 0.77, 0.62-0.96; $p=0.017$ ). LCA was not more predictive of perioperative complications compared to RPN (OR 1.63, 0.53-1.09 $\mathrm{p}=0.138$ ); and only RPN showed a reduction in complications with increasing procedure volume at the hospital (OR 0.71-0.40, $\mathrm{p}<0.05$ ).

\section{DISCUSSION}

RPN was initially described in 2004 and represents an acceptable alternative to open or laparoscopic PN for select localized renal tumors (17). It has been hypothesized that RPN may help overcome the technical challenges of LPN and may offer an easier transition to minimally invasive PN (18). In this retrospective study, we compared short-term perioperative outcomes of RPN vs. LCA, relying on a contemporary (2008-2010) population-based cohort of individuals undergoing minimally invasive treatment for localized renal tumors.

Our findings are several-fold. First, our contemporary analysis shows that RPN has similar blood loss and LOS compared to LCA, with reduced perioperative complications. In this nationally representative sample of inpatient admissions between October 2008 and December 2010, nearly three times as many RPNs were performed compared to LCAs (10,034 vs. 4,241), thus accounting for 70.3\% of treatment for this sample cohort. Although objective evidence has shown that the utilization of RPN has increased dramatically in recent years, no previous reports have identified this in the context of nephron sparing treatment (19).
Second, the characteristics of RPN vs. LCA cases differed regarding several patient and hospital attributes. However, on multivariate analysis, patients who were older and had a higher preoperative Charlson score were more likely to undergo LCA.

While treating, physicians might expect that older or sicker patients might benefit from LCA vs. RPN; we found that neither age nor treatment approach predicted complications. Despite the fact that there were no differences in complications, the groups that underwent the treatments were different. While we understand that there are patient and physician factors that may have influenced a patient to undergo a specific treatment beyond preoperative comorbidities, our study is similar to other publications which have shown that preoperative comorbidities are the most significant predictor of perioperative complication, and therefore all patients should be offered the treatment that gives the best oncologic outcome (20).

As a result of the increasing use of imaging techniques in the general population, there has been an increase in the detection rate of localized renal tumors.There is a general acceptance of nephron sparing approach as the first option in the management of a patient with an incidental localized renal tumors. Additionally it is accepted that the standard of care is still surgery due to its low level of postoperative complications and high oncologic efficacy $(7,21)$.

Although the increase in incidence of localized renal tumors has been seen among all age groups, it is most evident in the elderly populations who have more medical comorbidities. It is not surprising, therefore, that the potential application of minimally invasive treatment options for these incidental lesions has been of wide interest. Many retrospective studies have identified older age and preoperative comorbidity as predictors of receiving cryoablation, but these studies represent single centers and their conclusions could be influenced by sampling error and the surgeon's preferences within that institution (11).

While treating patients with localized renal tumors, three competing factors have to be balanced: cancer control, patient morbidity, and preservation of renal function (7). In examining 


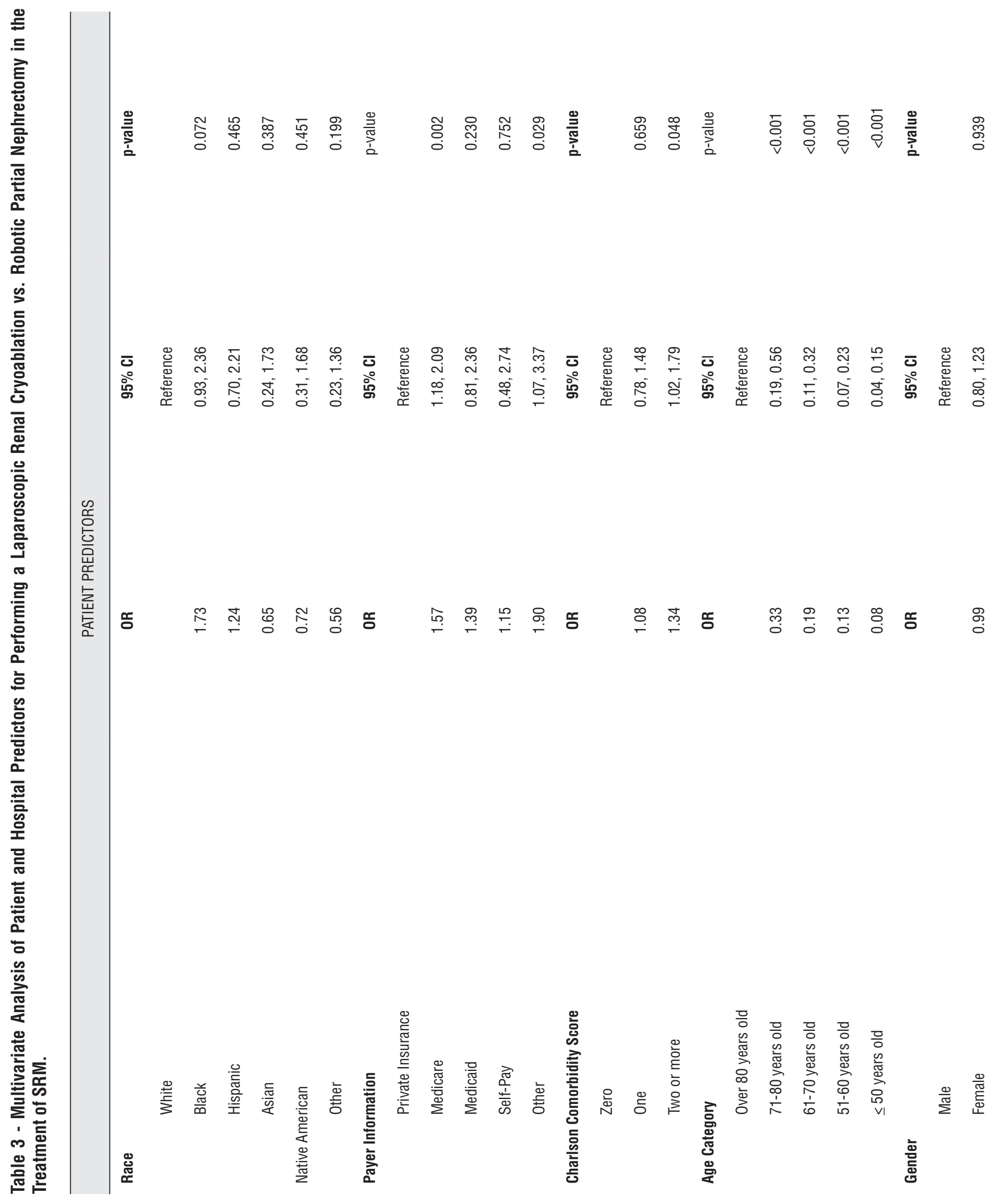




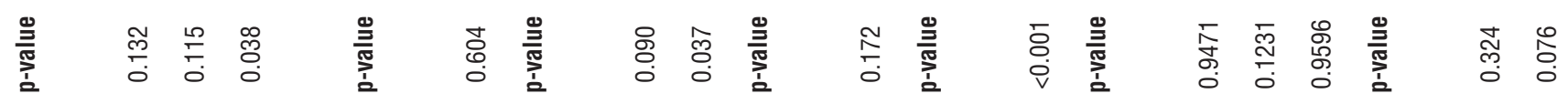

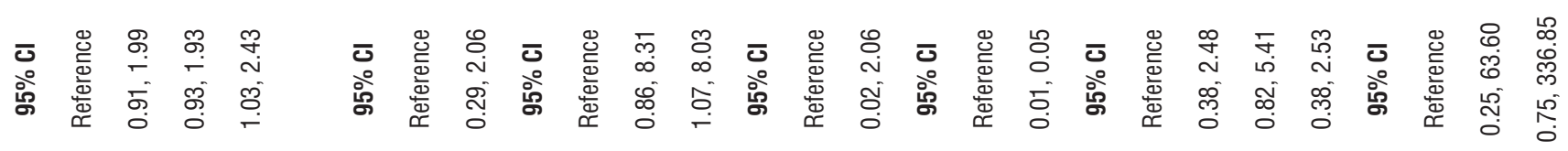

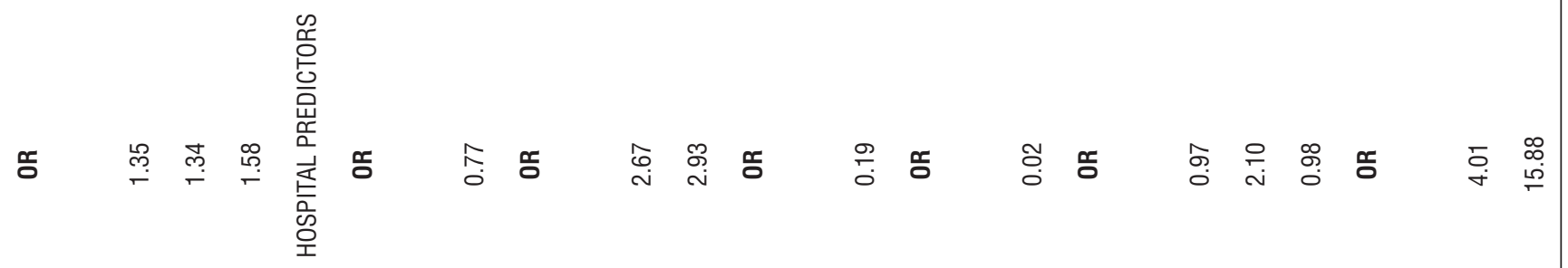

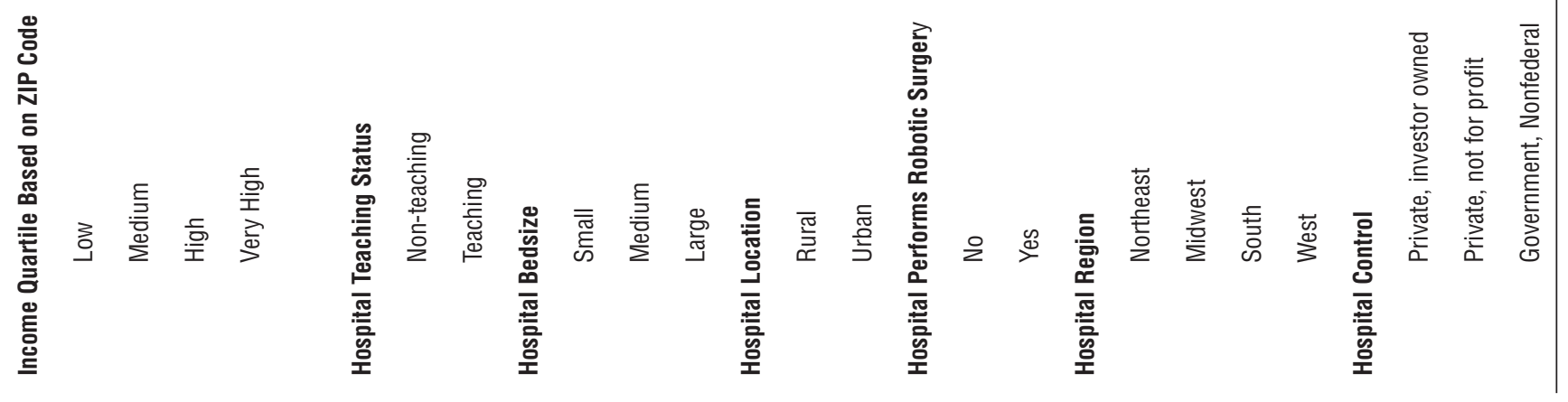




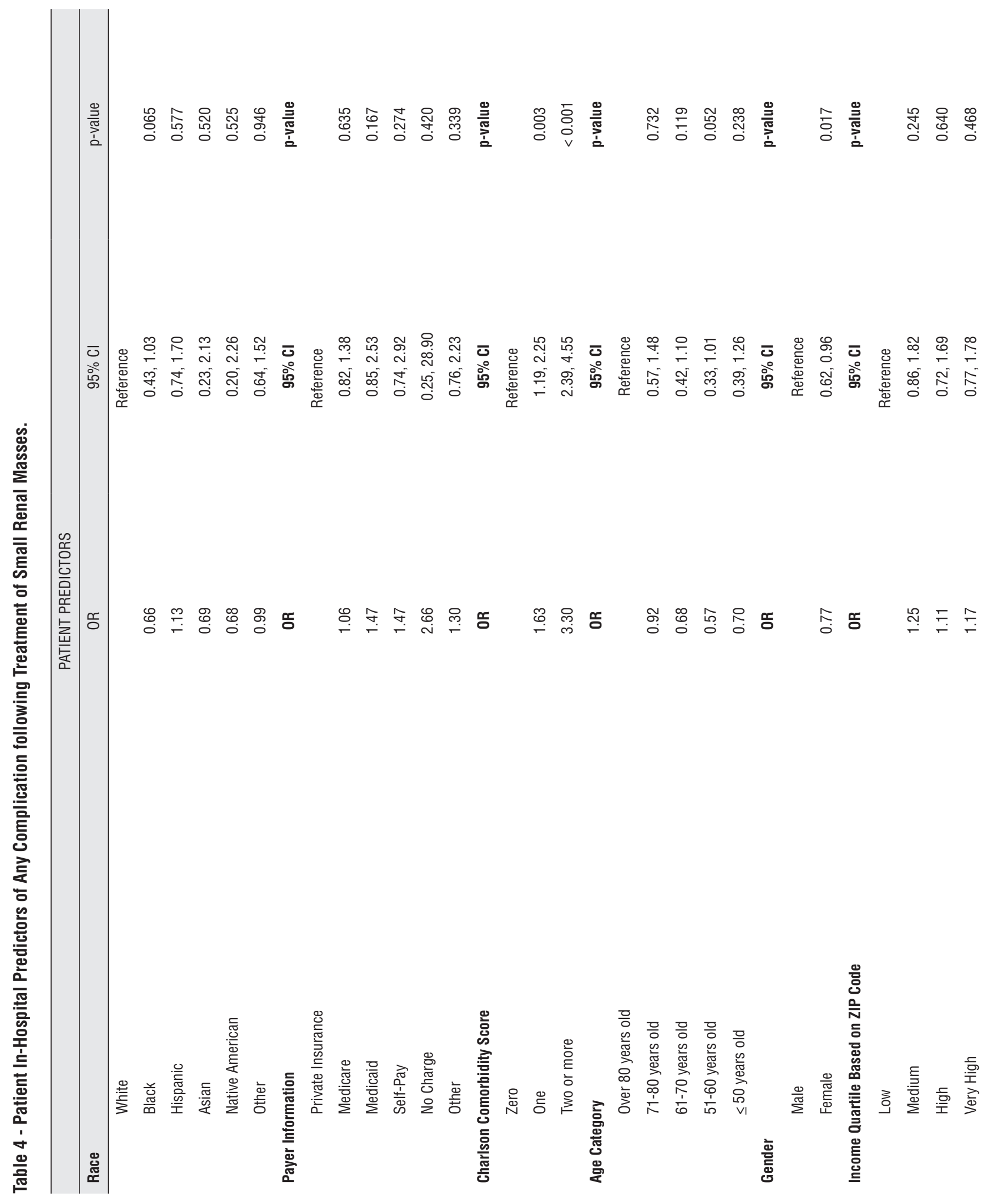




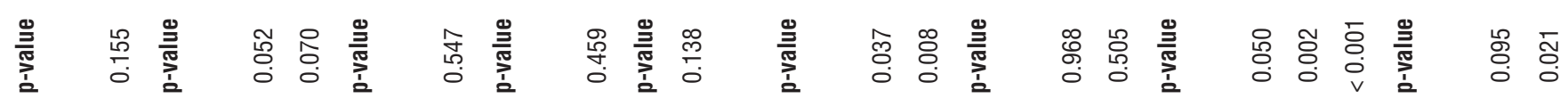

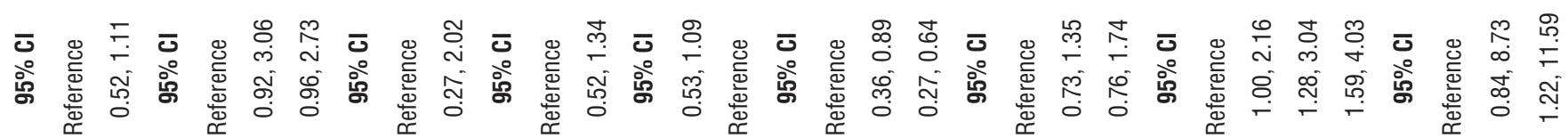

号

窟 
the advantages and disadvantages of the treatment and surgical options, only a few studies have formally compared ablation and extirpative surgery for localized renal tumors (22-24) and even fewer examining LCA and RPN $(25,26)$. The largest series from the Cleveland Clinic reported only 436 patients over a 12-year period with a mean follow-up of 4.8 months (27). A recent study by Emara et al. showed that with a mean follow-up of 16.5 months, there was no difference in perioperative measures (EBL or LOS) but LCA had a 3.6\% local recurrence rate vs. 0\% in RPN (9). This data is in line with a recent report by Klatte et al., who reported a higher recurrence rate in the LCA group compared with LPN and RPN group (28).

In the present series, there would appear to be no significant differences between the two treatment methods in terms of perioperative complications, blood transfusion, length of stay and cost. Our study was not able to assess any oncologic outcomes including positive surgical margins or local recurrence rate; however, a recent meta-analysis showed that no recurrence was detected in the RPN group, and had a positive surgical margin rate similar to other published series (27). Others have noted that RPN is a less challenging technique, compared to pure laparoscopic partial nephrectomy; specifically the shorter learning curve, minimal perioperative morbidity and shorter postoperative hospital stay $(8,29)$.

Nevertheless, our findings would support our general belief that RPN can truly challenge LCA; both procedures require general anesthesia, similar patient positioning and surgical incisions, and result in similar intra-operative and postoperative morbidity. As robotic systems presence in medical centers and surgeon experience with this platform continues to grow, the real question becomes clear: if patients are healthy enough to undergo general anesthesia, why should LCA be offered? Clearly, further and longer-term follow-up, preferably within a formal randomized study will be necessary to confirm oncologic and perioperative outcomes.

Several limitations of our study need to be considered. This analysis is affected by selection bias because of its retrospective design and because the NIS represents a large sample of patients with locali- zed renal tumors managed by RPN or LCA. Additionally, the NIS has no data on oncologic details such as stage, size, pathologic grade, atomic complexity, or margin status; additionally short and long term oncologic control is not reported in the NIS. Additionally we were unable to examine surgeon characteristics for the surgeries performed, as it is only complete for approximately half of the states in the NIS database, and therefore are unable to identify if the same surgeon or even practitioners from the same field (urology versus interventional radiology) performed these surgeries. And finally, NIS data is derived from hospital billing data based on ICD9 coding and not chart review and like any administrative database we cannot be sure of coding errors because this data was not confirmed by chart review.

\section{CONCLUSIONS}

Kidney tumors can be managed via a variety of techniques; for masses not amendable to percutaneous access for ablation, the choice is extirpative surgery or laparoscopic ablation.

Theoretically, LCA is a simpler surgical procedure than a RPN, which requires a hilar dissection, clamping of renal vessels, and renorrhaphy. However, our analysis of a large population-based cohort indicates that LCA does not appear to be associated with decreased complications compared to RPN. As studies suggest possible decreased oncologic control with CA compared to extirpative surgery, these findings call into question the utility of LCA as opposed to RPN in localized renal tumors not amenable to percutaneous ablation.

\section{CONFLICT OF INTEREST}

None declared.

\section{REFERENCES}

1. Miller DC, Ruterbusch J, Colt JS, Davis FG, Linehan WM, Chow $\mathrm{WH}$, et al. Contemporary clinical epidemiology of renal cell carcinoma: insight from a population based case-control study. J Urol. 2010;184:2254-8.

2. Mathew A, Devesa SS, Fraumeni JF Jr, Chow WH. Global increases in kidney cancer incidence, 1973-1992. Eur J Cancer Prev. 2002;11:171-8. 
3. Chow WH, Devesa SS, Warren JL, Fraumeni JF Jr. Rising incidence of renal cell cancer in the United States. JAMA. 1999;281:1628-31.

4. Rothman J, Egleston B, Wong YN, Iffrig K, Lebovitch S, Uzzo $R G$. Histopathological characteristics of localized renal cell carcinoma correlate with tumor size: a SEER analysis. J Urol. 2009;181:29-33; discussion 33-4.

5. Jayson M, Sanders $H$. Increased incidence of serendipitously discovered renal cell carcinoma. Urology. 1998;51:203-5.

6. Lane BR, Campbell SC, Gill IS. 10-year oncologic outcomes after laparoscopic and open partial nephrectomy. J Urol. 2013;190:44-9.

7. Campbell SC, Novick AC, Belldegrun A, Blute ML, Chow GK, Derweesh $\mathrm{IH}$, et al. Practice Guidelines Committee of the American Urological Association. Guideline for management of the clinical T1 renal mass. J Urol. 2009;182:1271-9.

8. Benway BM, Bhayani SB, Rogers CG, Dulabon LM, Patel MN, Lipkin M, et al. Stifelman MD. Robot assisted partial nephrectomy versus laparoscopic partial nephrectomy for renal tumors: a multi-institutional analysis of perioperative outcomes. J Urol. 2009;182:866-72.

9. Emara AM, Kommu SS, Hindley RG, Barber NJ. Robot-assisted partial nephrectomy vs laparoscopic cryoablation for the smal renal mass: redefining the minimally invasive 'gold standard'. BJU Int. 2014;113:92-9.

10. Kunkle DA, Uzzo RG. Cryoablation or radiofrequency ablation of the small renal mass : a meta-analysis. Cancer. 2008;113:267180.

11. Klatte T, Grubmüller B, WaldertM, WeibIP, Remzi M. Laparoscopic cryoablation versus partial nephrectomy for the treatment of small renal masses: systematic review and cumulative analysis of observational studies. Eur Urol. 2011;60:435-43.

12. Sammon JD, Karakiewicz PI, Sun M, Ravi P, Ghani KR, Jeong W, et al. Robot-assisted vs. laparoscopic partial nephrectomy: utilization rates and perioperative outcomes. Int Braz J Urol. 2013;39:377-86.

13. Anderson JE, Chang DC, Parsons JK, Talamini MA. The first national examination of outcomes and trends in robotic surgery in the United States. J Am Coll Surg. 2012;215:107-14; discussion 114-6.

14. Charlson ME, Pompei P, Ales KL, MacKenzie CR. A new method of classifying prognostic comorbidity in longitudinal studies: development and validation. J Chronic Dis. 1987;40:373-83.

15. Deyo RA, Cherkin DC, Ciol MA. Adapting a clinical comorbidity index for use with ICD-9-CM administrative databases. J Clin Epidemiol. 1992;45:613-9.

16. Hu JC, Gu X, Lipsitz SR, Barry MJ, D’Amico AV, Weinberg AC, et al. Comparative effectiveness of minimally invasive vs open radical prostatectomy. JAMA. 2009;302:1557-64.

17. Gettman MT, Blute ML, Chow GK, Neururer R, Bartsch G, Peschel R. Robotic-assisted laparoscopic partial nephrectomy: technique and initial clinical experience with DaVinci robotic system. Urology. 2004;64:914-8.
18. Sukumar S, Rogers CG. Robot-assisted partial nephrectomy. J Endourol. 2011;25:151-7.

19. Baillargeon-Gagné S, Jeldres C, Lughezzani G, Sun M, Isbarn H, Capitanio $U$, et al. A comparative population-based analysis of the rate of partial vs radical nephrectomy for clinically localized renal cell carcinoma. BJU Int. 2010;105:359-64.

20. Dindo D, Demartines N, Clavien PA. Classification of surgical complications: a new proposal with evaluation in a cohort of 6336 patients and results of a survey. Ann Surg. 2004;240:20513.

21. Ljungberg B, Cowan NC, Hanbury DC, Hora M, Kuczyk MA, Merseburger AS, et al. European Association of Urology Guideline Group. EAU guidelines on renal cell carcinoma: the 2010 update. Eur Urol. 2010;58:398-406.

22. Heuer R, Gill IS, Guazzoni G, Kirkali Z, Marberger M, Richie JP, et al. A critical analysis of the actual role of minimally invasive surgery and active surveillance for kidney cancer. Eur Urol. 2010;57:223-32.

23. Leveridge MJ, Mattar K, Kachura J, Jewett MA. Assessing outcomes in probe ablative therapies for small renal masses. J Endourol. 2010;24:759-64.

24. Stroup SP, Kopp RP, Derweesh IH. Laparoscopic and percutaneous cryotherapy for renal neoplasms. Panminerva Med. 2010;52:331-8.

25. Permpongkosol S, Bagga HS, Romero FR, Sroka M, Jarrett TW, Kavoussi LR. Laparoscopic versus open partial nephrectomy for the treatment of pathological T1NOM0 renal cell carcinoma: a 5-year survival rate. J Urol. 2006;176:1984-8; discussion 19889.

26. Gill IS, Matin SF, Desai MM, Kaouk JH, Steinberg A, Mascha $\mathrm{E}$, et al. Comparative analysis of laparoscopic versus open partial nephrectomy for renal tumors in 200 patients. J Urol. 2003;170:64-8.

27. Guillotreau J, Haber GP, Autorino R, Miocinovic R, Hillyer $S$, Hernandez A,et al. Robotic partial nephrectomy versus laparoscopic cryoablation for the small renal mass. Eur Urol. 2012:61:899-904.

28. Klatte T, Mauermann J, Heinz-Peer G, Waldert M, Weibl P, Klingler $\mathrm{HC}$, et al. Perioperative, oncologic, and functional outcomes of laparoscopic renal cryoablation and open partial nephrectomy: a matched pair analysis. J Endourol.2011;25:991-7.

29. Kural AR, Atug F, Tufek I, Akpinar H. Robot-assisted partial nephrectomy versus laparoscopic partial nephrectomy: comparison of outcomes. J Endourol. 2009;23:1491-7.

Correspondence address: Aaron C. Weinberg, MD Department of Urology Herbert Irving Pavilion, 11th Floor 161 Fort Washington Ave New York, NY, 10032, USA

Fax: +1 212 305-5100 E-mail: aw2616@columbia.edu 